\title{
An Improved Design of the Engine Bench Test Tool for the Dual Mass Flywheel (DMF)
}

\author{
Fan Dong ${ }^{1, *}$, Hong Zhang ${ }^{1}$, Ben Wang ${ }^{2}$, Zeguo Du ${ }^{1}$ and XiaoYu Yang ${ }^{2}$ \\ ${ }^{1}$ Powertrain Development Department, Dongfeng Motor Corporation Technical Center, 430056, Wuhan, China \\ ${ }^{2}$ Manufacturing Technology Department, Dongfeng Passenger Vehicle Company, 430056, Wuhan, China
}

\begin{abstract}
At present, a lot of passenger cars and light trucks are equipped with the dual mass flywheel, which can decrease the non-uniformity of the indicated engine torque. In engine bench test, the secondary mass of the dual mass flywheel can't be fixed because there is no transmission system on the test bench. As a result, the secondary mass of the dual mass flywheel will have radial and circumferential movements, which may lead to a damage to the dual mass flywheel. This paper presents an improved design of the crafting tool for the dual mass flywheel to protect the secondary mass during the engine bench test. For the improved design, the cylinder body is used for the positioning of the crafting tool, and a spline shaft and a ball bearing of centripetal adjustment are chosen to fix the secondary mass. Through this tool, the dual mass flywheel engine can be tested on the test bench without a starting shaft, and the disassembling of the engine flywheel bolts during the hot test can be avoided.
\end{abstract}

\section{Introduction}

With the ever-increasing demand to improve noise, vibration and harshness $(\mathrm{NVH})$ in vehicles, the dual mass flywheel (DMF) becomes one of the most significant power train contributions to the vehicles NVH. The DMF's primary aim is to reduce rattle and boom at various driving conditions. In 1985, BMW 525E was the first to put DMF into production in automotive history. They used a non-lubricating damper consisting of 8 straight-line heavy spring outside. Because the structure had serious wear problems, the first DMF has a short service life. The first lubricated DMF was used around 1987, in which a spring was used as an external damper and the lubricating oil was stored in the groove of the spring. Because of the existence of lubricating oil, the life of the DMF was elevated obviously. The arc spring damper was introduced in 1989, which was a breakthrough of the DMF. Due to this technology, the cost of the DMF fell immediately and the resonance problems were also solved. With the progress of technology and the reduction of cost, the application of DMF becomes more and more popular ${ }^{[1-3]}$. Luk's DMF was produced and sold about 100 million units in $2015^{[4,5]}$. The DMF consists of two separate flywheels connected by an arc-spring for damping the engine oscillations, which is linked to the engine's crank shaft directly. The adjustment between masses and springdamper is determined in a way to realize a mechanical low pass filter to reduce the cyclic irregularity of the engine torque ${ }^{[6-9]}$. The DMF divides the single mass flywheel (SMF) into two parts. One part is retained at the side of the engine to act as the SMF for starting and transmitting the rotational torque of the engine, which is called the primary mass. The other part called the secondary mass is placed at the transmission side to increase the rotational inertia ${ }^{[10]}$.

The bench test is an important procedure of the engine production, which can detect effectively the leakage of the fuel, the water and the air as well as the abnormal sound ${ }^{[11]}$. During the bench test of the DMF engine, there is usually no transmission system on the test bench which is used to fix the secondary mass. The secondary mass will be jerked relative to the primary mass, which may lead to a damage to the DMF. Thus when the engine is equipped with the DMF, the common solution is to use the SMF for bench test and after the bench test completed, the SMF is replaced with the DMF. However, this way will increase the cost of manufacture and can't validate the DMF effectively. In order to realize the bench test of the DMF, a kind of crafting tool structure was developed by Anhui Jianghuai Automobile Group Corp (JAC) [12]. This company used a crafting tool to fix the secondary mass by four lengthened bolts. The crafting tool designed can guarantee the secondary mass rotating with the primary mass when the DMF is working, but it also needs to disassemble half of the flywheel bolts $(80-120 \mathrm{Nm})$. The JAC's design firstly realized the bench test of the DMF engine, but there are two shortages with this kind of design. The first one is that the DMF crafting tool can only be used on the bench test with a starting shaft. The second one is that the flywheel bolts must be disassembled during the bench test process. Until now, there is no case taken as example for the DMF engine without disassembly the flywheel bolts. In this paper, an improved design is provided for the bench test crafting tool for the DMF engine to overcome the shortcomings of the previous design. The bench test crafting tool developed comprises of three parts: tool body, ball bearing and spline shaft. The

Corresponding author: dongfan@dfmc.com.cn 
adjustment between the ball bearing inside the tool body and the spline shaft connected to the DMF is chosen to prevent the secondary mass swinging during the engine bench test.

The paper is organized as the following: the operational principle of DMF and the design scheme of the crafting tool is described in detail in Section 2. Section 3 presents the previous and the improved design of crafting tool for the DMF. Conclusions are provided in Section 4.

\section{Details about the design}

\subsection{The operational principle of DMF}

The DMF is generally mounted between the engine and the gearbox, and the engine crankshaft is connected to the primary mass of the DMF to drive the flywheel to rotate, and the secondary mass is suitable for the gearbox to transfer power ${ }^{[13-15]}$. The primary and the secondary masses are connected by the spring, and the torsional vibration can be eliminated by the counter-rotation of the two masses ${ }^{[16,17]}$. However, during the power transmission process, the vibration of engine may induce the disalignment between the engine crankshaft and the gearbox input shaft. This disalignment may lead to an extrusion deformation between the primary and the secondary masses, affect the NVH performance of the vehicle and even make damage to the DMF. At present, the DMF usually has a centrifugal pendulum structure, and the periodical swing of the pendulum mass makes it as a short-term energy storage device, which can eliminate the torsional vibration in the power transmission system and solve the above disalignment problems effectively. A centrifugal pendulum-type DMF comprises of flanges, multiple pendulum weights on flanges and connecting pins. The pendulum mass and the flange are comprised with grooves, and the connecting pins are equipped with the grooves so that the pendulum mass can centrifugally swing under the limitation of the grooves and the connecting pins ${ }^{[18,19]}$. The pendulum mass swings along the circumferential direction, and when it reaches the limiting position relative to the flange, the pendulum mass will contact with the damping component and then stop swinging. In this way, the impact and noise caused by the swing of the DMF in the circumferential position are weakened or eliminated. Figure 1 shows the schematic of the required position for the DMF crafting tool.

In the periodical swinging process of the pendulum mass relative to the flange, the damping component switches between the working and non-working states. There is a certain relationship between the central angle of the gap on the pendulum mass $\theta$ and the central angle of the bulge part $\delta$. As shown in Figure 1, the two limiting positions of the pendulum mass swing relative to the flange on circumferential periodically as $l_{l}$ and $l_{2}$. $l_{l}$ is the left limiting position of the pendulum mass swinging along the anti-clockwise, while $l_{2}$ is the right limiting position of the pendulum mass swinging along the clockwise. Between the two positions, the maximum rotation angle of the pendulum mass relative to the flange is $\beta$, thereinto the maximum angle that drives the damping component to rotate relative to the flange is $2 \gamma$. In Figure 1 define the free angle as $\alpha$. The region corresponding to the free angle $\alpha$ is not covered by the trajectory of the pendulum mass relative to the flange when the pendulum mass swings periodically. Therefore, if the central angle of the bulge part $\delta$ is smaller than the free angle $\alpha$, the pendulum mass will not touch the damping component, and then the damping component will not be in working state and have no buffer function. While if the central angle of the bugle part $\delta$ is too large, the non-working state of the damping component will be too small or even no space, which will hinder the normal centrifugal swing of the pendulum mass. In practical applications, the recommended value of $\gamma$ is about $2^{\circ}$, and $\delta$ is slightly larger than $\alpha$. Moreover, the central angle of the gap on the pendulum mass gap $\theta$ needs to be slightly larger than the central angle of the bulge part $\delta$, so that the displacement of the pendulum mass is approximately in the middle of the two limiting positions of the circumferential oscillation, which will not be impeded by the damping component. That is to say, at the condition $\delta<\theta-\beta / 2$, the pendulum mass can operate smoothly in the working process of the $\mathrm{DMF}^{[20]}$.

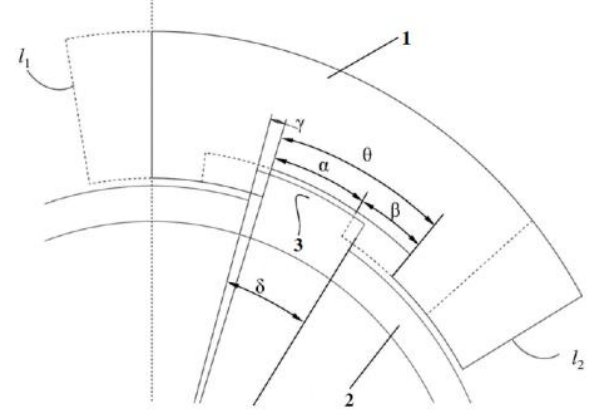

Figure 1. The schematic of the required position for the DMF crafting tool (1-the pendulum mass, 2-the damping component,

3 -the bulge part, $\alpha$-the free angle, $\beta$-the maximum rotation angle of the pendulum mass relative to the flange, $\gamma-1 / 2$ of the maximum rotation angle of the damping component relative to the flange, $\delta$-The central angle of the bulge part, $\theta$-the central

angle of the gap on the pendulum mass gap, $l_{l}$-the limiting

axial swing position of the pendulum mass along anticlockwise, $l_{2}$-the limiting axial swing position of the pendulum mass along clockwise)

\subsection{The design scheme of the crafting tool}

In view of the DMF operational principle, two directions of constraint needs to be satisfied in order to achieve the DMF bench test. Firstly, the DMF with a pendulum mass should ensure the secondary mass circumferential movement meet the requirement of the damping component, as shown in Figure 1. In fact, the condition $\delta<\theta-\beta / 2$ is very difficult to meet in practice, and an alternative way that fixed the secondary mass in the primary mass is considered in this paper. Secondly, during the operation process of the DMF, if the engine crankshaft and the gearbox input shaft are disalignment, there will be a radial clearance between the primary and the secondary masses. When the radial clearance is large 
enough, there may be an internal force in the DMF that may result in a damage to the DMF. Hence, the crafting tool must confirm the secondary mass has no radial displacement during the bench test and the circumferential swing meet the working range of the centrifugal pendulum structure.

At present, a spline shaft is used to connect the engine crankshaft with the secondary mass so that the secondary mass can be driven when the engine crankshaft drives the primary mass. When the DMF is working on the test bench, the gears of the spline shaft engage with the gears of the secondary mass to ensure the primary and secondary masses without relative movement in circumferential and radial directions. Figure 2 shows the structure of the crafting tool for the DMF at present. The crafting tool developed in this paper consists of three parts: tool body, ball bearing and spline shaft. The tool body is comprised with two connecting plates, which is connected with the cylinder body by four fixed bolts. The connecting plates are also equipped with positioning ring for localization during the installation. The core components of the crafting tool are the spline shaft and the ball bearing. The coordination of the spline shaft and ball bearing drives the secondary mass to run in an appropriate space in the process of the bench test operation. The existence of the ball bearing can ensure that the tool body doesn't rotate when the DMF is working. At last, in order to reduce the weight of the crafting tool and the labor intensity effectively, the crafting tool is chosen to be made of aluminum alloy and 26 weight-reducing holes are also added on the tool body.

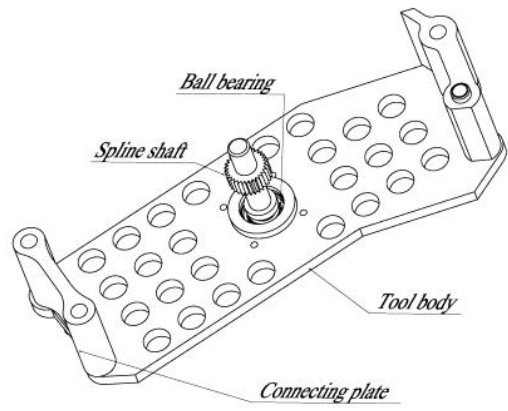

Figure 2. The structure of the crafting tool for the DMF in present

\section{Results and discussion}

\subsection{The previous design of crafting tool for the DMF}

In order to realize the bench test of the DMF engine, few papers has been carried out on the DMF engine bench test tool. Figure 3 shows a kind of crafting tool structure developed by Anhui Jianghuai Automobile Group Corp (JAC). The JAC's crafting tool comprises of the tool body and the lengthened fixing bolts. The tool body is provided with the installation surface which is pressed on the outer surface of the secondary mass of the DMF. Four installation holes are also equipped on the tool body, and the lengthened fixing bolts pass through the installation holes mentioned above, the through-hole of the primary and secondary masses and are fixed on the engine crankshaft firmly. The four installation holes are preferably chosen as counterbore so that the lengthened fixing bolts are completely located in the installation holes to prevent the interference between the lengthened fixing bolts and the surrounding parts during the bench test. Specifically, the JAC's DMF engine normally has 8 flywheel bolts. Half of them are used to fix the flywheel on the engine crankshaft before the bench test, and the other 4 flywheel bolts will be replaced by 4 lengthened bolts to fix the tool body to prevent the axial movement of the secondary mass and the relative rotation between the primary and secondary masses during the bench test. In addition, in the JAC's structure, an installation hole is also provided to fix the starting shaft of the bench test, which is applicable to the central starting mode test bench. The installation holes of the bench test starting shaft are designed as spline holes, and the starting shaft is fixed on the tool body by the spline. Because the tool body is meshed with the secondary mass by the starting shaft of the test bench, the crafting tool will rotate with the DMF, thus ensuring the DMF isn't damaged during the bench test. After the engine bench test, the 4 lengthened fixing bolts and the crafting tool are removed, and 4 flywheel bolts are replaced to fix the DMF for regular working of the engine. The crafting tool developed by the JAC is the first time to realize the bench test of the DMF, and the process of the DMF replacement is also cancelled by the optimized design. However, this design is only applicable to the engine test bench with a starting shaft, while the engine test benches in most production lines have no starting shafts. For this type of test bench, the test engine needs to start up with the self-carried starter, so the JAC's design can't be used in this method. Meanwhile, for this kind of crafting tool, 4 flywheel bolts $(80-120 \mathrm{Nm})$ still need to be disassembled, which leads to a high labor intensity.

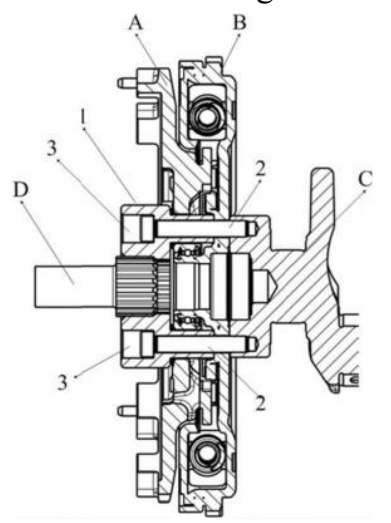

Figure 3. The structure of crafting tool for the DMF by JAC (A-the secondary mass, B-the primary mass, C-engine crankshaft, D-starting shaft of the test bench, 1-tool body, 2lengthened fixing bolts, 3 -installition through-hole)

\subsection{The improved design scheme}

In view of the JAC's design, the radial, axial and circumferential directions of the secondary mass are totally restricted on the primary mass. Actually, according to the principle of the DMF, there is no need to restrict the axial movement of the secondary mass. 
Therefore, in order to avoid the assembly and dismounting of the flywheel bolts, the cylinder body is chosen to position the crafting tool in the present improved design. The crafting tool can be connected with the secondary mass of the DMF through the ball bearing in the center, so that only the spline shaft rotates with the DMF when the flywheel is working, while the crafting tool does not rotate. Figure 4 shows the assembly position of the crafting tool for the DMF. As shown in Figure 4, the DMF crafting tool developed in the present study comprises of three parts: tool body, spline shaft and fixing bolts. There is a spline shaft hole in the center of the tool body, and both sides of the tool body are provided with connecting plate. The connecting plate is provided with four installation holes through which the tool body is fixed on the engine by the fixing bolts, and the end surface of the tool body is the mounting surface that fit with the cylinder body. At the same time, a centripetal adjustment ball bearing is also arranged in the center position of the tool body. The spline shaft passes through the installation hole and connects with the tool body through the centripetal adjustment ball bearing.

During the engine bench test, one end of the spline shaft is extended to the crankshaft of the engine and the other end is into the centripetal adjustment ball bearing. The gear in the spline shaft is completely meshed with the secondary mass gear of the DMF, and the engagement between the gear of the spline shaft and the secondary mass is chosen to realize the fixing of the secondary mass on the bench test. The four installation holes on the tool body are aligned on the installation holes on the cylinder body respectively, and then the tool body is fixed on the cylinder body by the fixing bolts. Through this method, the crafting tool can prevent the relative rotation between the primary and secondary masses of the DMF during the engine working, and the damage to the DMF caused by the abnormal relative motion between the two masses can also be avoided effectively. Moreover, due to no need to replace the SMF for the bench test, the crafting tool is good for the cost saving and the performance of the DMF can be detected better.

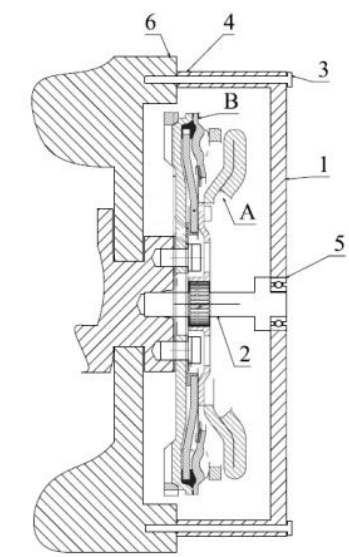

Figure 4. The assembly position of the crafting tool for the DMF at present (A-the secondary mass, B-the primary mass, 1tool body, 2-spline shaft, 3-fixing bolts, 4-connecting plate, 5ball bearing, 6-cylinder body)
According to the improved design, the engine crafting tool for the DMF can work efficiently in bench test. Figure 5 shows the actual picture of the crafting tool and the installation position. In practice, a position ring is installed on the tool body for positioning in the installation process. Meanwhile, the centripetal adjustment ball bearing in the center of the tool body is connected with the tool body through the connection block. A groove is also equipped on the center of the connection block for the centripetal adjustment ball bearing, and the outside part of the centripetal adjustment ball bearing is fixed in the center of the tool body through a clamping spring. The connection block is unnecessary to be installed when the crafting tool is using. During the engine bench test, the stress on the tool body is small, and it is often not necessary to install all 4 lengthened fixing bolts. Generally only 2 diagonally bolts are needed to further reduce the workload.
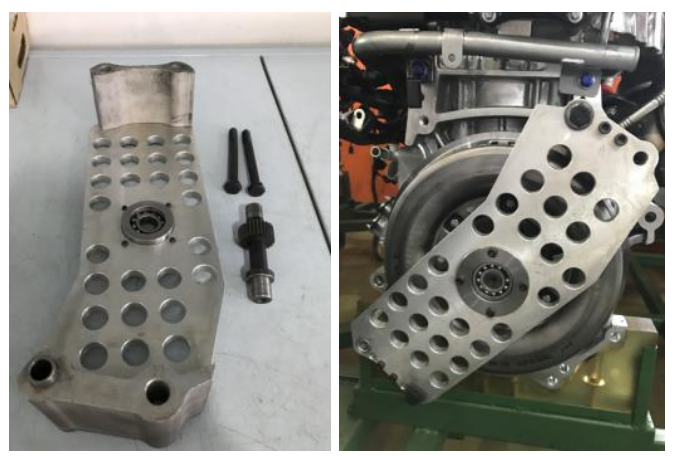

Figure 5. The actual picture of the DMF crafting tool and installation position

\section{Conclusions}

In this paper, an improved designed engine bench test crafting tool was developed for the DMF engine. Compared with the previous design, the improved design mainly optimizes the following aspects. Firstly, the improved design can be used on the test bench without a starting shaft. By connecting the spline shaft with the secondary mass, the test engine can be started by the starter carried by itself during the bench test. Secondly, the improved design eliminates the restriction on the axial movement. In the improved design, only the circumferential and radial movements of the DMF are restricted, and due to reduction of the restriction conditions, the fixture does not depend on the crafting tool itself for positioning. At last, the improved design can be used without removing the flywheel bolts. Because the crafting tool uses the cylinder body for positioning, only 2 positioning bolts are needed to tighten on the cylinder body. The secondary mass can be fixed by the spline shaft, which can avoid disassembly of the flywheel bolts. Through these methods, the improved design has achieved excellent results, and the production efficiency is improved obviously.

\section{References}

1. L.P. Zeng, L.Q. Song, J.D. Zhou. Mech Mach Theory. 92(2015). 
2. Y.L. Wang, X.P. Qin, S. Huang, S. Deng. Appl Acoust. 104(2016).

3. Y.J. Lee, T.Y. Shim, K.O. Lee, M.D. Hur, S.S. Kang. J Mech Sci Technol. 27, 8(2013).

4. G. Pica, C. Cervone, A. Senatore, M. Lupo, F. Vasca. Intell Ind Syst. 2, 2(2016).

5. J.J. Oh, S.B. Choi. IEEE-ASME T Mech. 20, 1(2015).

6. S. Lin, S. Chang, B. Li. Mechatronics. 24, 8(2014).

7. G.A. Timofeev, E.O. Podchasov, Int. J. Mech. Eng. Robot. Res. 9, 3(2020).

8. T. Kodama, Y. Honda, Int. J. Mech. Eng. Robot. Res. 9, 7(2020).

9. M. Pisaturo, A. Senatore. Appl Therm Eng. 93 (2016).

10. S.S. Hiremath, I.G. Bhavi, Int. J. Mech. Eng. Robot. Res. 3, 4(2014).

11. A. Khamlichi, M. Bezzazi, M.A.P. Vera. J Mater Process Tech. 142,3(2003).
12. S.S. Chen, L.Q. Yang, Y.B. Zhang etc. China, CN201620348228.4,2016.

13. Z. Zhao, H. Chen, Q. Wang. Math Probl Eng. 2014.

14. J. Popelka, Int. J. Mech. Eng. Robot. Res. 9, 1(2020)

15. H.C. Terán, G.R. Torres, O. Arteaga, J.J. Morales, B.P. León, D.A. Morales, Int. J. Mech. Eng. Robot. Res. 8, 4(2019).

16. L. Chong, R. Zeng, Z Jiang. Adv Mech Eng. 7,6(2015).

17. R. Grega, J. Krajňák, P. Baran. Geophys J Int. 128, 2(2014)

18. C.L. Tang, X.J. Dai, X.Z. Zhang, L. Jiang. J Mech Sci Technol. 26, 9(2012).

19. D. Qin, Y. Liu, J. Hu, R. Chen. J Mech Eng. 46, 18(2010).

20. Y. Wang, Y. Li, N. Li, H. Sun, C. Wu, T. Zhang. P I of Mech Eng. 228, 5 (2014). 